\title{
Cartesian Lattice Counting by the Vertical 2-sum
}

\author{
Jukka Kohonen ${ }^{1}$ \\ Received: 8 July 2020 / Accepted: 10 May 2021 / Published online: 25 May 2021
}

(C) The Author(s) 2021

\begin{abstract}
A vertical 2-sum of a two-coatom lattice $L$ and a two-atom lattice $U$ is obtained by removing the top of $L$ and the bottom of $U$, and identifying the coatoms of $L$ with the atoms of $U$. This operation creates one or two nonisomorphic lattices depending on the symmetry case. Here the symmetry cases are analyzed, and a recurrence relation is presented that expresses the number of nonisomorphic vertical 2-sums in some desired family of graded lattices. Nonisomorphic, vertically indecomposable modular and distributive lattices are counted and classified up to 35 and 60 elements respectively. Asymptotically their numbers are shown to be at least $\Omega\left(2.3122^{n}\right)$ and $\Omega\left(1.7250^{n}\right)$, where $n$ is the number of elements. The number of semimodular lattices is shown to grow faster than any exponential in $n$.
\end{abstract}

Keywords Counting · Vertical 2-sum · Modular lattice $\cdot$ Distributive lattice

\section{Introduction}

Let $L$ and $U$ be finite lattices. Their vertical sum is obtained by identifying the top of $L$ with the bottom of $U$. If $L$ has two coatoms and $U$ has two atoms, their vertical 2-sum is obtained by removing the top of $L$ and the bottom of $U$, and identifying the coatoms of $L$ with the atoms of $U$.

The vertical sum leads to a simple and well-known recurrence relation. A lattice is a vilattice (short for vertically indecomposable) if it is not a vertical sum of two non-singleton lattices. If $f(n)$ and $f_{\mathrm{vi}}(n)$ are the numbers of nonisomorphic $n$-element lattices and vilattices, respectively, then

$$
f(n)=\sum_{k=2}^{n} f_{\mathrm{vi}}(k) f(n-k+1), \quad \text { for } n \geq 2 .
$$

We call this Cartesian counting because each term expresses the cardinality of a Cartesian product, namely, of the set of $k$-element vi-lattices and the set of $(n-k+1)$-element lattices.

Jukka Kohonen

jukka.kohonen@iki.fi

1 Department of Mathematics and Systems Analysis, Aalto University, Espoo, Finland 
One does not need to list the members of a Cartesian product to find its cardinality. The recurrence in Eq. 1 has been used in counting small lattices [1-5] and in proving lower bounds $[1,6]$.

Many vi-lattices can be further decomposed as vertical 2-sums of smaller lattices. So let us use this decomposition to pursue a kind of Cartesian counting of vi-lattices. Now we must observe that from two given lattices, one obtains two vertical 2-sums, because there are two ways to match the coatoms and the atoms. Whether the results are isomorphic depends on the symmetries of $L$ and $U$.

Our main result, Theorem 1, is a recurrence relation that distinguishes the symmetry cases, and expresses the exact number of nonisomorphic lattices obtainable as vertical 2sums, in terms of the numbers of component lattices. The new relation is analogous to Eq. 1 but works with the vertical 2-sum. To apply it we need to classify and count the component lattices by symmetry type.

The motivations of this study are threefold. First, our recurrence provides a new way of counting small lattices. Only the component lattices are generated explicitly; their vertical 2 -sums are then counted with the recurrence in the Cartesian fashion. It is relatively easy to restrict methods of generating modular and distributive lattices so that they only generate the components, and leave out all vertical 2-sums. This is faster, so we can count further. We count modular and distributive vi-lattices of at most 35 and 60 elements, respectively. This also verifies previous countings (of at most 33 and 49 elements), because the method is different.

The second motivation is a more compact lattice listing. A full listing of distributive vilattices of at most 60 elements would contain about $4.9 \times 10^{12}$ lattices. We can shrink the list to less than $1 / 200$ of that size, to $2.3 \times 10^{10}$ lattices, by leaving out all vertical 2 -sums. A smaller listing is more practical to store and to study, and if desired, one can still recover the left-out lattices by performing vertical 2-sums on the listed components.

The third motivation is in improving lower bounds. A simple recurrence for vertical 2sums was derived in [6], but it is only a loose lower bound as it does not consider the symmetry cases. The new recurrence gives tighter bounds because of an extra factor of 2 in the asymmetric cases. It may not sound like much, but the factor compounds when vertical 2 -sum is applied repeatedly. Some further improvement comes from counting small lattices larger than before. For modular vi-lattices we improve the lower bound from $\Omega\left(2.1562^{n}\right)$ [6] to $\Omega\left(2.3122^{n}\right)$. For distributive vi-lattices we improve from $\Omega\left(1.678^{n}\right)$ [1] to $\Omega\left(1.7250^{n}\right)$, which is close to the empirical growth rate.

\section{Vertical 2-sum and Symmetry}

In order to understand how the vertical 2-sum operates on lattices, we classify them by the number and symmetry of their atoms and coatoms. Our aim is in modular and distributive vi-lattices, but we state the results more generally when convenient. All lattices considered in this work are finite. If $L$ is a lattice, we write $a(L)$ and $c(L)$ for the numbers of its atoms and coatoms, and $0_{L}$ and $1_{L}$ for its bottom and top.

Definition 1 Let $L$ and $U$ be disjoint lattices of length 3 or greater, such that $L$ has two coatoms $c_{1}, c_{2}$ and $U$ has two atoms $a_{1}, a_{2}$. Then their vertical 2 -sums are the two lattices obtained by removing $1_{L}$ and $0_{U}$, and identifying $\left(c_{1}, c_{2}\right)$ with either $\left(a_{1}, a_{2}\right)$ or $\left(a_{2}, a_{1}\right)$. We say that $L$ and $U$ are the summands of the vertical 2-sum. 
Note that vertical 2-sums are indeed lattices, those of graded lattices are graded, and those of vi-lattices are vi-lattices [6]. We do not consider summands of length 2 as that would be essentially an identity operation. If $S$ is a vertical 2-sum of $L$ and $U$, then $|S|=|L|+|U|-4$.

Definition 2 If a lattice has two coatoms [atoms], they are symmetric if the lattice has an automorphism that swaps them, and fixed otherwise.

Lemma 1 Let $L$ and $U$ be lattices with vertical 2-sums $S_{1}$ and $S_{2}$. Then $S_{1}$ and $S_{2}$ are nonisomorphic if and only if $L$ has fixed coatoms and $U$ has fixed atoms.

Proof If $L$ has an automorphism that swaps the coatoms, then extending it with the identity mapping on $U$ yields an isomorphism $S_{1} \rightarrow S_{2}$. If $U$ has symmetric atoms, the case is similar. Finally, if there is an isomorphism $S_{1} \rightarrow S_{2}$, it must either fix the coatoms of $L$ and swap the atoms of $U$, or vice versa; but this is impossible if $L$ has fixed coatoms and $U$ has fixed atoms, so in that case $S_{1}$ and $S_{2}$ are nonisomorphic.

So far we have allowed both graded and nongraded lattices. For the rest of this work, we will require the vertical 2-sums and their summands to be graded. This is sufficient for our main concern (modular and distributive lattices), and makes it easy to define an inverse operation of the vertical 2-sum, namely, decomposition into a lower and upper summand such that the upper summand is not itself a vertical 2-sum. This decomposition is uniquely determined by locating the highest-ranked non-coatom two-element level (if one exists). It might be possible to extend this idea to the nongraded case and still ensure unique decomposition, but we sidestep such issues with our restriction.

We also restrict our attention to vertically indecomposable lattices. After we have counted those, we can easily use Eq. 1 to include vertically decomposable lattices in the counts. So, we are now concerned with graded vi-lattices, and we divide them into three kinds as follows.

Definition 3 If $L$ is a graded vi-lattice, then its $k$ th level, denoted $L_{k}$, is the set of elements that have rank $k$. A neck is a two-element level other than the atoms and the coatoms. We say that $L$ is:

1. a composition, if it contains a neck;

2. a piece, if does not contain a neck, has rank 3 or greater, and at least one of $a(L)$ and $c(L)$ equals two;

3. special otherwise.

A composition has necessarily at least 8 elements, and a piece has at least 6 . It is clear that all compositions ensue from pieces by repeated application of the vertical 2-sum. Specials are not vertical 2-sums, but also cannot act as their summands, because they are too short (rank two or smaller) or contain too many atoms and coatoms.

Definition 4 A piece $L$ is:

1. a middle piece, if $a(L)=c(L)=2$;

2. a bottom piece, if $a(L) \geq 3$ and $c(L)=2$;

3. a top piece, if $a(L)=2$ and $c(L) \geq 3$. 
A middle piece can act as either summand of a vertical 2-sum. A bottom piece can act only as the lower summand, and a top piece only as the upper summand. Pieces are further divided into symmetry types as follows (see also Fig. 1).

Definition 5 A middle piece is of symmetry type:

1. MF, if its atoms and coatoms are fixed;

2. MA, if its atoms are symmetric and coatoms are fixed;

3. MC, if its atoms are fixed and coatoms are symmetric;

4. MX, if it has an automorphism that swaps the atoms but fixes the coatoms, and another automorphism that swaps the coatoms but fixes the atoms;

5. $\mathrm{MH}$, if it is not $\mathrm{MX}$, but has an automorphism that swaps both the atoms and the coatoms.

Note that in an MX piece, atoms and coatoms can be swapped independently, but in an $\mathrm{MH}$ piece only simultaneously. The shapes of the letters $\mathrm{X}$ and $\mathrm{H}$ are meant as mnemonics for this (think of the center of $\mathrm{X}$ as a freely rotating joint). The distinction between MX and $\mathrm{MH}$ will be essential when building larger lattices.

Definition 6 A bottom piece is of symmetry type:

1. BF, if its coatoms are fixed;

2. BS, if its coatoms are symmetric.

Definition 7 A top piece is of symmetry type:

1. TF, if its atoms are fixed;

2. TS, if its atoms are symmetric.

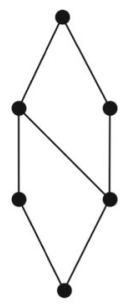

$\mathrm{MF}$

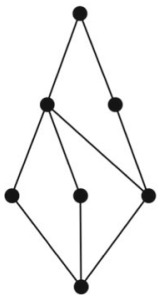

$\mathrm{BF}$

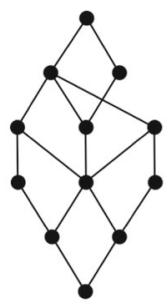

MA

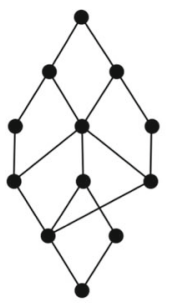

$\mathrm{MC}$

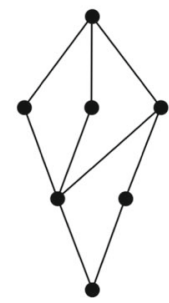

TF

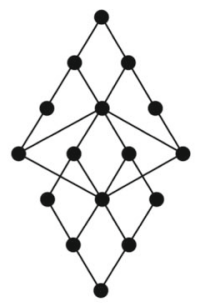

MX

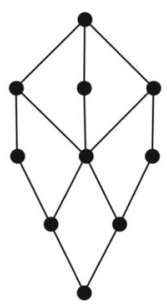

TS

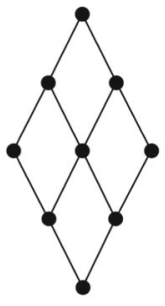

$\mathrm{MH}$

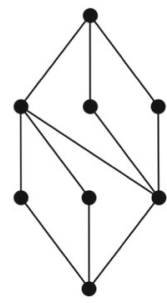

special

Fig. 1 Example pieces of each symmetry type (and a special) 
The example pieces in Fig. 1 are small for illustration purposes, but generally pieces can be much larger: in our enumerating tasks some pieces will have more than 50 elements. We will form compositions "bottom up". A vertical 2-sum of two pieces is a composition. Given a composition, adding a piece on top yields one or two bigger compositions. To keep track of the number and symmetry of the results, we need to define symmetry types for compositions too.

Definition 8 A composition is of symmetry type:

1. CF, if it has two coatoms and they are fixed;

2. CS, if it has two coatoms and they are symmetric;

3. CN ("composition-nonextensible"), if it has three or more coatoms.

For example, if we take the BF piece from Fig. 1 as the lower summand, and the MC piece as the upper summand, we obtain two nonisomorphic vertical 2-sums of type CS, as shown in Fig. 2. More generally, the following lemma characterizes how many and what kinds of compositions are formed.

Lemma 2 If $L$ is a piece or a composition, and $U$ is a piece, then the number and type of their nonisomorphic vertical 2-sums are as follows.

\begin{tabular}{llllllll}
\hline & \multicolumn{7}{c}{ U type } \\
\cline { 2 - 8 } L type, any of & $\mathrm{MF}$ & $\mathrm{MA}$ & $\mathrm{MC}$ & $\mathrm{MX}$ & $\mathrm{MH}$ & $\mathrm{TF}$ & $\mathrm{TS}$ \\
\hline $\mathrm{CF} / \mathrm{BF} / \mathrm{MF} / \mathrm{MA}$ & $2 \times \mathrm{CF}$ & $1 \times \mathrm{CF}$ & $2 \times \mathrm{CS}$ & $1 \times \mathrm{CS}$ & $1 \times \mathrm{CF}$ & $2 \times \mathrm{CN}$ & $1 \times \mathrm{CN}$ \\
$\mathrm{CS} / \mathrm{BS} / \mathrm{MC} / \mathrm{MX} / \mathrm{MH}$ & $1 \times \mathrm{CF}$ & $1 \times \mathrm{CF}$ & $1 \times \mathrm{CS}$ & $1 \times \mathrm{CS}$ & $1 \times \mathrm{CS}$ & $1 \times \mathrm{CN}$ & $1 \times \mathrm{CN}$ \\
$\mathrm{CN} / \mathrm{TF} / \mathrm{TS}$ & none & none & none & none & none & none & none \\
\hline
\end{tabular}

Proof Let us first prove the numbers. We proceed by the rows.

On the first row, $L$ has two fixed coatoms. By Lemma 1, if $U$ has fixed atoms (MF, MC or TF), then there are two nonisomorphic vertical 2-sums; otherwise there is one.
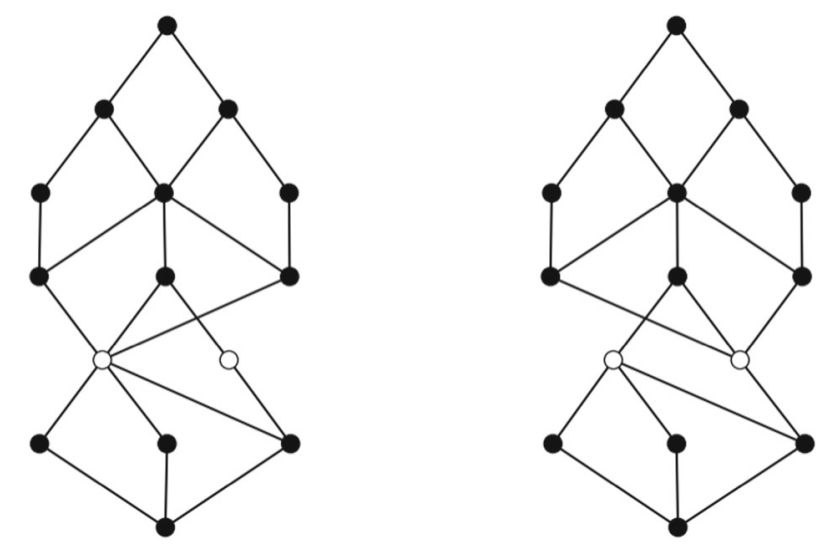

Fig. 2 The two vertical 2-sums of a BF piece and an MC piece, with hollow circles indicating the neck where the summands connect 
On the second row, $L$ has two symmetric coatoms. By Lemma 1 there is one vertical 2-sum up to isomorphism.

On the third row, $L$ has 3 or more coatoms, so no vertical 2 -sums are formed.

Next we deduce the symmetry type of each vertical 2-sum $S_{i}(i=1,2)$. We proceed by the columns.

If $U$ is MF or MA, it has fixed coatoms. Then $S_{i}$ cannot have an automorphism that swaps the coatoms of $S_{i}$, because its restriction to $U$ would be an automorphism that swaps the coatoms of $U$. Thus $S_{i}$ has fixed coatoms.

If $U$ is MC or MX, it has an automorphism that swaps its coatoms and fixes its atoms; extending with the identity mapping in $L$ gives, in each $S_{i}$, an automorphism that swaps the coatoms. Thus $S_{i}$ has symmetric coatoms.

If $U$ is $\mathrm{MH}$, then an automorphism of $S_{i}$ that swaps the coatoms also swaps the atoms of $U$, which are also the coatoms of $L$. Thus $S_{i}$ has symmetric coatoms if and only if $L$ has symmetric coatoms.

If $U$ is TF or TS, then $S_{i}$ has three or more coatoms and is of type $\mathrm{CN}$.

\section{Cartesian Counting in a Lattice Family}

In this section we present our main result, a recurrence relation that counts all nonisomorphic compositions in some desired lattice family, provided that the family has suitable form. We also give examples of such families.

Definition 9 A family $\mathcal{F}$ of graded vi-lattices is (vertically) 2-summable if the following conditions hold:

(C1) If $L, U \in \mathcal{F}$ and $S$ is one of their vertical 2-sums, then $S \in \mathcal{F}$.

(C2) If $S \in \mathcal{F}$ is a vertical 2-sum of $L$ and $U$, then $L, U \in \mathcal{F}$.

The first condition ensures that vertical 2-sums stay in the family, and the second ensures that all compositions in $\mathcal{F}$ are indeed obtained as vertical 2 -sums of smaller lattices in $\mathcal{F}$.

Theorem 1 Let $\mathcal{F}$ be a 2-summable family, and let $\mathrm{XX}_{n}$ denote the number of nonisomorphic n-element lattices in $\mathcal{F}$ having symmetry type $X X$. Then for $n<8$ we have $\mathrm{CF}_{n}=\mathrm{CS}_{n}=\mathrm{CN}_{n}=0$, and for $n \geq 8$ the following recurrences hold:

$$
\begin{aligned}
\mathrm{CF}_{n} & =\sum_{j=6}^{n-2}\left(\mathrm{LF}_{j} \cdot\left(2 \cdot \mathrm{MF}_{k}+\mathrm{MA}_{k}+\mathrm{MH}_{k}\right)+\mathrm{LS}_{j} \cdot\left(\mathrm{MF}_{k}+\mathrm{MA}_{k}\right)\right) \\
\mathrm{CS}_{n} & =\sum_{j=6}^{n-2}\left(\mathrm{LF}_{j} \cdot\left(2 \cdot \mathrm{MC}_{k}+\mathrm{MX}_{k}\right)+\mathrm{LS}_{j} \cdot\left(\mathrm{MC}_{k}+\mathrm{MX}_{k}+\mathrm{MH}_{k}\right)\right) \\
\mathrm{CN}_{n} & =\sum_{j=6}^{n-2}\left(\mathrm{LF}_{j} \cdot\left(2 \cdot \mathrm{TF}_{k}+\mathrm{TS}_{k}\right)+\mathrm{LS}_{j} \cdot\left(\mathrm{TF}_{k}+\mathrm{TS}_{k}\right)\right)
\end{aligned}
$$

where $k=n-j+4$, and

$$
\begin{aligned}
& \mathrm{LF}_{j}=\mathrm{CF}_{j}+\mathrm{BF}_{j}+\mathrm{MF}_{j}+\mathrm{MA}_{j} \\
& \mathrm{LS}_{j}=\mathrm{CS}_{j}+\mathrm{BS}_{j}+\mathrm{MC}_{j}+\mathrm{MX}_{j}+\mathrm{MH}_{j} .
\end{aligned}
$$


Proof For $n<8$ the claim holds because compositions have at least 8 elements.

Let then $n \geq 8$ and consider an $n$-element CF-type composition $S \in \mathcal{F}$. There is exactly one way of expressing $S$ as a vertical 2-sum of two lattices $L, U$ such that $U$ is a piece. This $U$ contains the elements of $S$ above and including its highest-ranked neck, plus an augmented bottom element. By condition (C2) we have $L, U \in \mathcal{F}$. Furthermore, because $|U| \geq 6$ and and $|L|+|U|-4=n$, it follows that $|L| \leq n-2$.

We also observe that different nonisomorphic choices of $L$ and $U$, where $U$ is a piece, lead to nonisomorphic results. To be more precise: If $L \not L^{\prime}$ or $U \not U^{\prime}$, and $U$ and $U^{\prime}$ are pieces, then the vertical 2-sums of $L$ and $U$ are isomorphic to the vertical 2-sums of $L^{\prime}$ and $U^{\prime}$.

All nonisomorphic $n$-element CF-type compositions in $\mathcal{F}$ can be counted by considering (for all $j=6, \ldots, n-2$ ) first the choices of a $j$-element lower summand $L \in \mathcal{F}$, and then the choices of an upper summand $U \in \mathcal{F}$ such that $U$ is a piece with $k=n-j+4$ elements, subject to the requirement that the resulting vertical 2-sums are of type CF.

Now $\mathrm{LF}_{j}$ is the number of nonisomorphic lower summands that have fixed coatoms. For each such lower summand, by collecting the CF-type results from the first row of the table in Lemma 2, we get $2 \cdot \mathrm{MF}_{k}+\mathrm{MA}_{k}+\mathrm{MH}_{k}$ nonisomorphic vertical 2-sums, which are in $\mathcal{F}$ by condition $(\mathrm{C} 1)$.

Similarly, $\mathrm{LS}_{j}$ is the number of nonisomorphic lower summands that have symmetric coatoms. For each such lower summand, by collecting the CF-type results from the second row of the table in Lemma 2, we get $\mathrm{MF}_{k}+\mathrm{MA}_{k}$ nonisomorphic vertical 2-sums, which are in $\mathcal{F}$ by condition $(\mathrm{C} 1)$.

Adding up the cases we obtain the stated expression for $\mathrm{CF}_{n}$. The expressions for $\mathrm{CS}_{n}$ and $\mathrm{CN}_{n}$ follow in the same manner.

Not all families of graded vi-lattices are 2-summable. For a simple example, finite graded rank-four vi-lattices fail both conditions $(\mathrm{C} 1)$ and $(\mathrm{C} 2)$. Interestingly, finite geometric lattices are 2-summable but in a vacuous way.

Theorem 2 The only finite geometric lattice that has a two-element level is $M_{2}$.

Proof Let $L$ be a finite geometric lattice that has a two-element level $\{a, b\}$. Because $L$ is atomistic, neither $a$ nor $b$ has any join-irreducible covers, thus $a$ and $b$ are each covered by exactly one element $c$. Further, because $L$ is necessarily vertically indecomposable, it follows that $c=1_{L}$, and $a, b$ are the coatoms.

The numbers of atoms and coatoms in a finite geometric lattice of rank $r$ are known as the Whitney numbers $W_{1}$ and $W_{r-1}$. It is known that $W_{1} \leq W_{r-1}$; see e.g. Dowling and Wilson [7]. This implies that our $L$ has two atoms. But we have shown that $L$ cannot have two-element levels other than the coatoms; thus the atoms are the coatoms, and $L=$ $M_{2}$.

In other words, Theorem 2 says that all finite geometric lattices are special; there are no pieces and no compositions, so no use for the vertical 2-sum. Modular and distributive lattices will be more interesting for our purposes. We first prove an auxiliary result by elementary means.

Lemma 3 In the Hasse diagram of a finite semimodular lattice, the subgraph induced by two consecutive levels is connected. 
Proof Let $L$ be a finite semimodular lattice, $L_{k}$ its $k$ th level, and $H_{k}$ the subgraph induced by $L_{k}$ and $L_{k+1}$. We use induction on $k$. Clearly $H_{0}$ is connected. Assume then that $H_{k-1}$ is connected. If $L_{k}$ is a singleton, then obviously $H_{k}$ is connected. Otherwise, let $(U, V)$ be any partition of $L_{k}$ into two nonempty subsets. Because $H_{k-1}$ is connected, there is an element in $L_{k-1}$ that is covered by some two elements $u \in U$ and $v \in V$. Then by semimodularity $u$ and $v$ are covered by some $w \in L_{k+1}$, so there is a path from $U$ to $V$ in $H_{k}$. Finally, from every element in $L_{k+1}$ there is an edge to $L_{k}$. Thus $H_{k}$ is connected.

Note that Lemma 3 also follows from previously known, more general results: Björner [8] proved that finite semimodular lattices are lexicographically shellable, and Collins [9] proved that graded lexicographically shellable lattices are rank-connected (i.e. the subgraph induced by two consecutive levels is connected).

Lemma 4 The family of finite semimodular vi-lattices is 2-summable.

Proof We use subscripted symbols $\wedge_{L}, \vee_{L}$ and $\prec_{L}$ to denote meet, join and covered-by in lattice $L$.

(C1) Let $L$ and $U$ be finite semimodular vi-lattices, $S$ their vertical 2-sum, and $N$ the two identified elements of $L$ and $U$. Clearly $S$ is a vi-lattice. We show that $S$ is semimodular by using Birkhoff's condition [10, p. 331]. Let $a, b \in S$ such that they cover $a \wedge_{S} b$. Then $a$ and $b$ have the same rank, and are either below $N$, in $N$ or above $N$. If $a, b$ are below $N$, then because $L$ is semimodular, $a, b \prec_{S} a \vee_{L} b=$ $a \vee_{S} b$. If $a, b$ are in or above $N$, then because $U$ is semimodular, $a, b \prec_{S} a \vee_{U} b=$ $a \vee_{S} b$.

(C2) Let $S$ be a finite semimodular vi-lattice that is a vertical 2-sum of $L$ and $U$. Clearly $L$ and $U$ are vi-lattices. We show that they are semimodular, again using Birkhoff's condition.

First let $a, b \in L$ such that they cover $a \wedge_{L} b$. If $a, b$ are not the coatoms of $L$, then $a \vee_{L} b=a \vee_{S} b$. If $a, b$ are the coatoms of $L$, then $a \vee_{L} b=1_{L}$. In both cases $a, b \prec_{L} a \vee_{L} b$. Thus $L$ is semimodular.

Let then $a, b \in U$ such that they cover $a \wedge_{U} b$. If $a, b$ are not the atoms of $U$, then $a \wedge_{U} b=a \wedge_{S} b$, and because $S$ is semimodular, $a, b \prec_{U} a \vee_{U} b=a \vee_{S} b$. If $a, b$ are the atoms of $U$, then they are a neck of $S$. Because $S$ is semimodular, it follows from Lemma 3 that $a, b$ have a common upper cover $c$ in $S$. Then also $a, b \prec_{U} c$. Thus $U$ is semimodular.

Lemma 5 The family of finite modular vi-lattices is 2-summable.

Proof Follows from Lemma 4 by duality.

Lemma 6 The family of finite distributive vi-lattices is 2-summable.

Proof We recall that a finite modular lattice is distributive if and only if it does not contain a cover-preserving diamond [10, p. 109], that is, five distinct elements $o, a, b, c, i$ such that $o \prec a, b, c \prec i$.

(C1) Let $L$ and $U$ be finite distributive vi-lattices and $S$ their vertical 2-sum. By Lemma 5 $S$ is modular. Since $L$ and $U$ do not contain a cover-preserving diamond, the only possibility for $S$ to contain one would be with $o \in L$ and $i \in U$, but the neck 
consisting of the two identified elements of $L$ and $U$ cannot contain three distinct elements $a, b, c$. Thus $S$ is distributive.

(C2) Let $S$ be a finite distributive vi-lattice that is a vertical 2-sum of $L$ and $U$. By Lemma $5 L$ and $U$ are modular vi-lattices. Since $S$ does not contain a coverpreserving diamond, the only possibility for $L$ to contain one would be with $i=1_{L}$, but this is impossible because $L$ has only two coatoms. Thus $L$ is distributive. The case of $U$ is similar.

\section{Computations}

\subsection{Method of Classifying a Lattice}

Given a graded vi-lattice represented by its covering graph, a short piece of program code classifies the lattice into the types described in Section 2. Calculating lattice length, counting atoms and coatoms, and finding possible necks is straightforward. For analyzing the symmetry type we use Nauty version $2.7 \mathrm{r} 1[11,12]$.

Nauty returns the automorphism group of a given directed graph as a list of generators $\left(\gamma_{1}, \ldots, \gamma_{k}\right)$. To classify a bottom piece we check if any generator swaps the coatoms; in that case the coatoms are symmetric, otherwise fixed. With a top piece we check if any generator swaps the atoms. To classify a middle piece some more cases are required. For each generator, we check if it:
A. swaps the atoms and fixes the coatoms; or
B. swaps both the atoms and the coatoms; or
C. swaps the coatoms and fixes the coatoms.

Generators that touch neither atoms nor coatoms are ignored. Then:

1. If there are no generators of types $\mathrm{A} / \mathrm{B} / \mathrm{C}$, the piece is $\mathrm{MF}$.

2. If there are generators of type $A$, but none of $B / C$, the piece is MA.

3. If there are generators of type $\mathrm{C}$, but none of $\mathrm{A} / \mathrm{B}$, the piece is $\mathrm{MC}$.

4. If there are generators of type $\mathrm{B}$, but none of $\mathrm{A} / \mathrm{C}$, the piece is $\mathrm{MH}$.

5. If there are generators of at least two of the types $\mathrm{A} / \mathrm{B} / \mathrm{C}$, the piece is $\mathrm{MX}$.

It is easily seen that this procedure produces the correct classification. Note that for an MX piece, Nauty does not necessarily return generators of types A and C. It can instead return, for example, a generator $\gamma_{i}$ of type A, and a generator $\gamma_{j}$ of type B. But then $\gamma_{i} \circ \gamma_{j}$ is an automorphism that swaps the coatoms and fixes the atoms, and then we know that the piece is indeed MX.

\subsection{Modular Lattices}

Modular vi-lattices were previously generated and counted up to 30 elements in [5], and up to 33 elements in unpublished work [13]. That was done with a program that starts from length-two seed lattices, and then adds new levels of elements recursively. The program lists exactly one representative lattice from each isomorphism class.

We use here essentially the same lattice-generating program, modified so that it skips all compositions, and generates only the pieces and the specials. The modification is simply that two-element levels are not allowed between coatom and atom levels, because such a level would form a neck. 
With this program, all modular pieces and specials of $n \leq 35$ elements were generated (up to isomorphism), and classified as described in Section 4.1. The numbers of modular compositions of $n \leq 35$ elements were then calculated using Lemma 5 and Theorem 1 .

The results of the exact counting are shown in Table 1. Rows MF-TS and "special" are from direct counting with the lattice-generating program. Rows $\mathrm{CF}-\mathrm{CN}$ are calculated with the recurrence in Theorem 1. Row "vi-latt." contains the numbers of all modular vi-lattices: this is the sum of specials, pieces and compositions. Finally, row "all" has the numbers of all modular lattices (including vertical sums of vi-lattices), calculated with the vertical sum recurrence in Eq. 1.

An exponential lower bound is derived as follows. Using Theorem 1 with the known numbers of modular pieces of up to 35 elements, and plugging in zeros for larger pieces (whose numbers we do not know), we obtain lower bounds on $\mathrm{CF}_{n}, \mathrm{CS}_{n}$ and $\mathrm{CN}_{n}$ for $n$ arbitrarily large. We observe that the growth ratios (from $n$ to $n+1$ ) of all three bounds settle a little above 2.3122 for $n$ large enough. To obtain rigorous lower bounds, we choose a convenient starting point $n=50$, convenient constant coefficients in front, and apply induction.

Theorem 3 The numbers of nonisomorphic modular compositions of types CF, CS and CN have the following lower bounds, when $n \geq 50$ :

$$
\begin{aligned}
\mathrm{CF}_{n} & \geq 0.002910 \times 2.3122^{n} \\
\mathrm{CS}_{n} & \geq 0.000035 \times 2.3122^{n} \\
\mathrm{CN}_{n} & \geq 0.002470 \times 2.3122^{n}
\end{aligned}
$$

Proof For $50 \leq n \leq 85$ the claim follows by direct calculation with Theorem 1, using the numbers of pieces from Table 1, and zeros when the number of pieces is not known.

For $n>85$ the claim follows by induction on $n$. Let $n>85$ be arbitrary, and assume that the claimed lower bounds hold on $\mathrm{CF}_{m}, \mathrm{CS}_{m}$ and $\mathrm{CN}_{m}$ when $n-35 \leq m \leq n-1$. Then applying Theorem 1 gives the claimed lower bounds on $\mathrm{CF}_{n}, \mathrm{CS}_{n}$ and $\mathrm{CN}_{n}$, which completes the induction.

Corollary 1 There are at least $0.005415 \times 2.3122^{n}$ nonisomorphic modular vi-lattices of $n$ elements when $n \geq 50$.

Proof Add up the three lower bounds from Theorem 3.

Theorem 4 There are at least $0.02 \times 2.3713^{n}$ nonisomorphic modular lattices of $n$ elements when $n \geq 100$.

Proof For $100 \leq n \leq 400$ the claim follows by direct calculation. First use Theorem 1 to compute the exact numbers of modular vi-lattices for $n \leq 35$, and lower bounds for $35<n \leq 400$ by replacing the unknown piece counts with zeros. Then apply Eq. 1 to compute lower bounds on modular lattices.

For the induction step, let $n>400$ be arbitrary, and assume that the claimed lower bound holds for the previous 300 values. Applying Eq. 1 then gives the claimed lower bound for the number of $n$-element modular lattices. This completes the induction. 


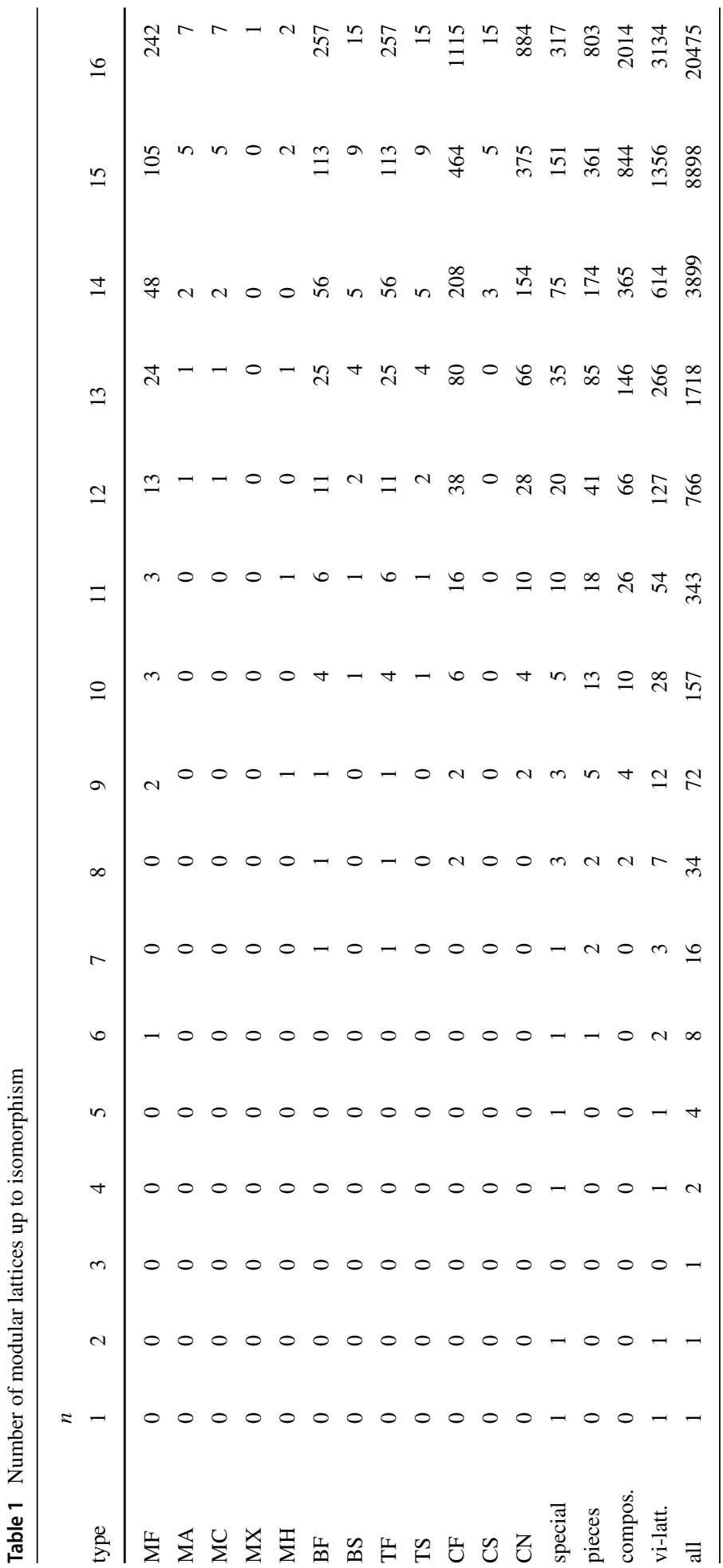




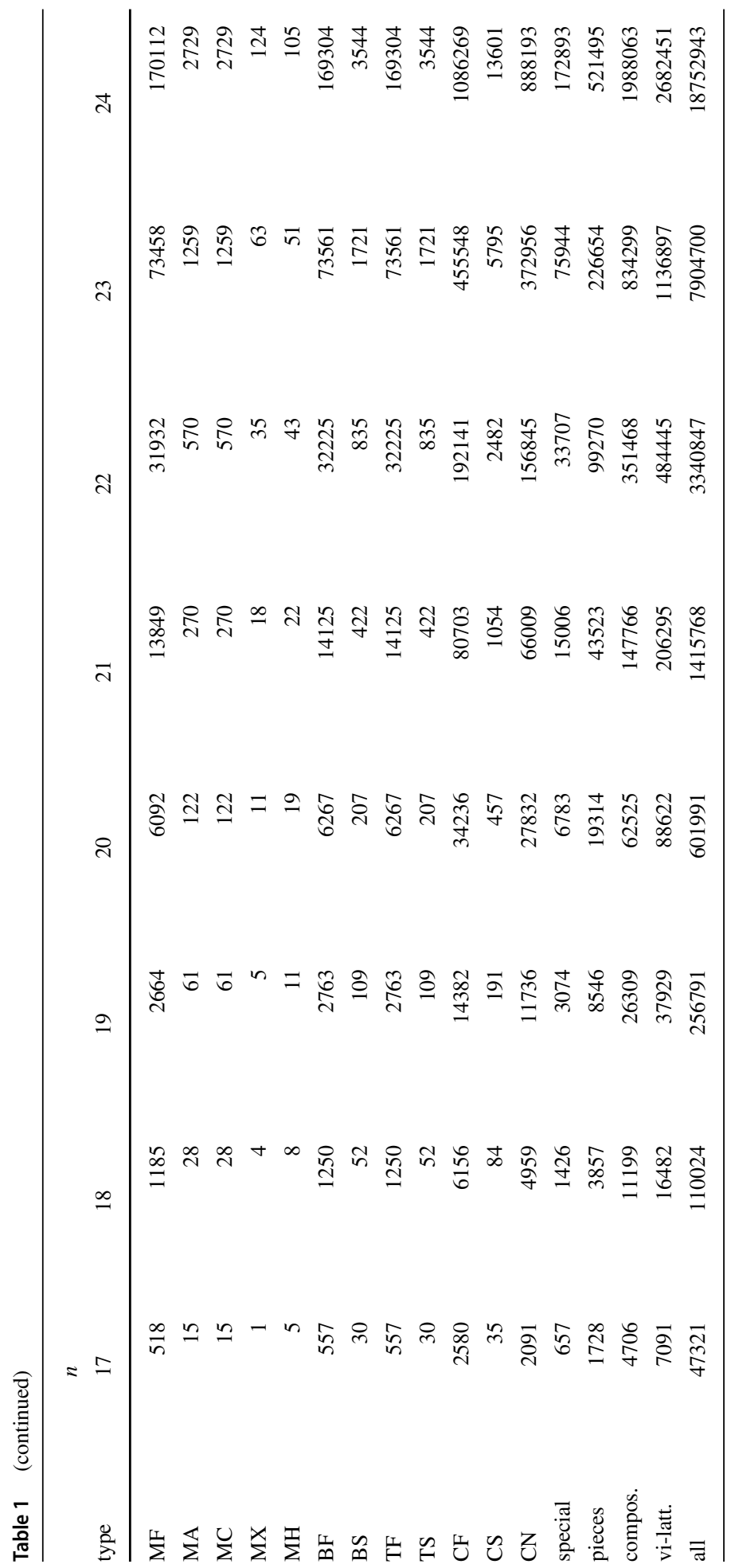




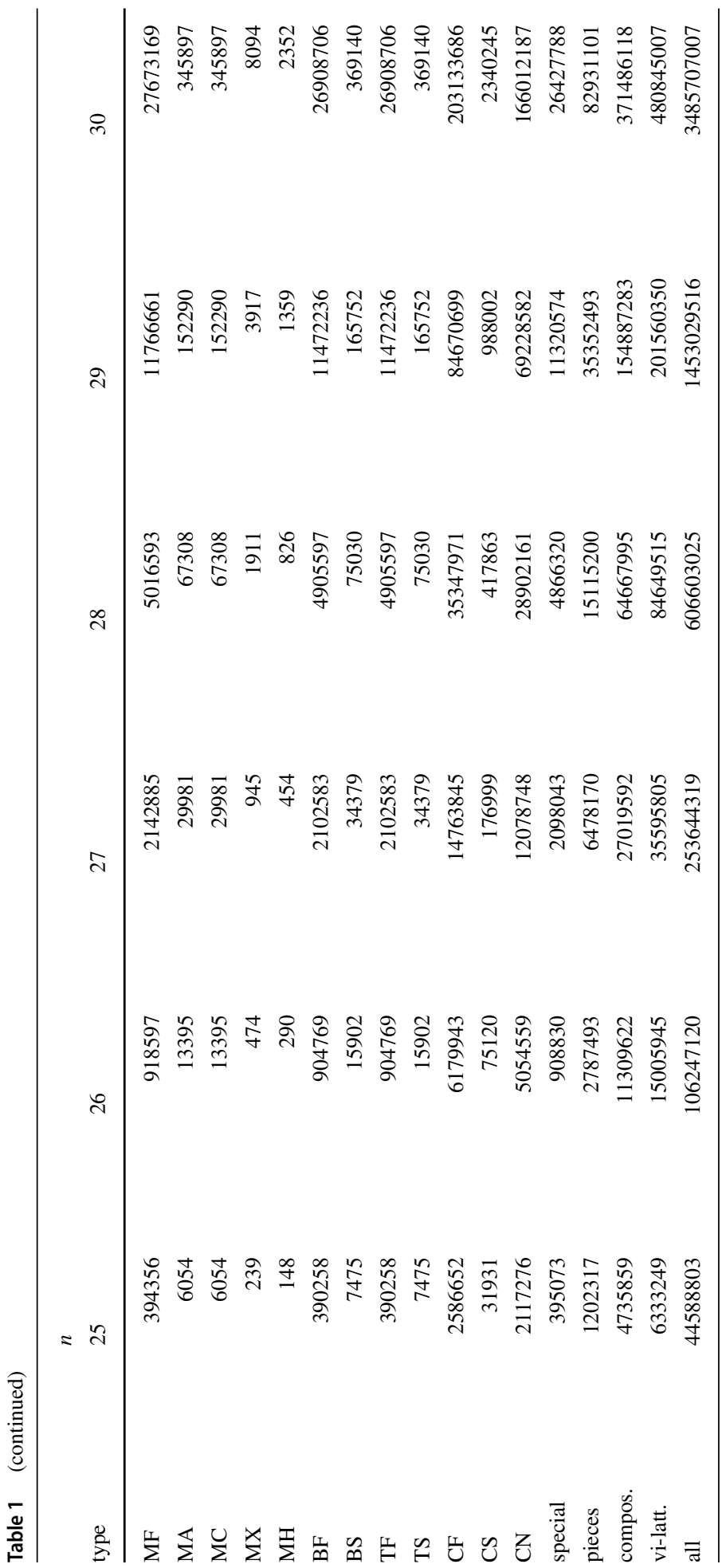




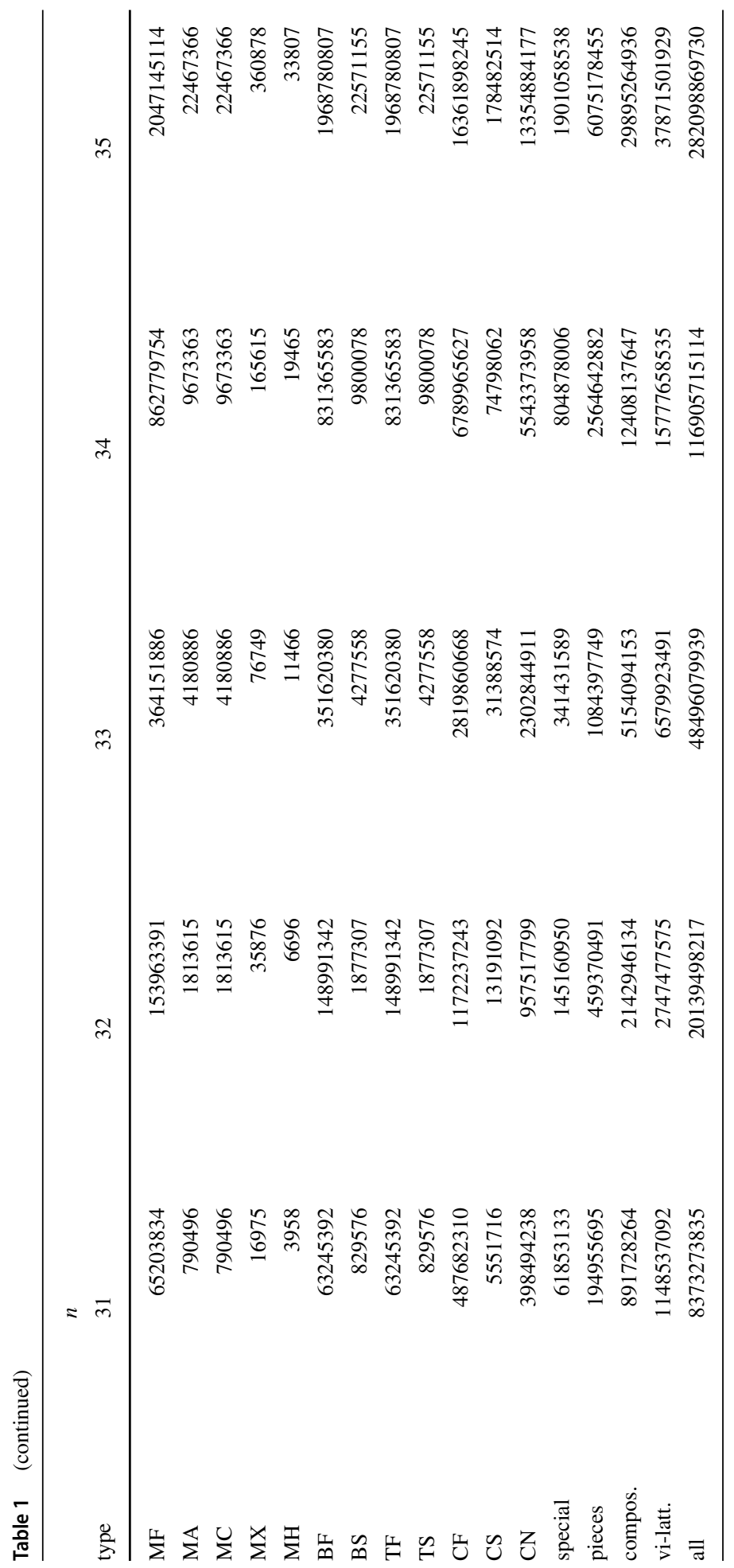


The accompanying SageMath code [14] demonstrates how the lower bounds can be verified by calculation: see functions paper_lower_bound_verify_modular and paper_v1_lower_bound_verify_modular.

\subsection{Distributive Lattices}

Distributive vi-lattices have been previously counted up to 49 elements by Erné et al. [1, 15]. To count $n$-element distributive lattices, they actually generated posets that have $n$ antichains; these are in a one-to-one correspondence with the distributive lattices.

Our approach is more direct. We generate the distributive lattices directly, using the same program that we used for modular lattices, with some modifications. The first modification is a condition that ensures that we generate only the distributive lattices. Since the original program generates modular lattices, we only need to check that whenever a new element is created, it does not create a cover-preserving diamond [10, p. 109]. This ensures that we generate the distributive lattices but no others.

We also employ an important optimization that cuts short search branches that cannot lead to distributive lattices. Our lattice-generating program (see [5] for more details) builds lattices levelwise, top down, until the number of elements reaches a preset maximum. When creating a new level, it adds new elements in decreasing order of updegree. The last step on each level is thus to create meet-irreducible elements. In the original algorithm, this step can create a large number of meet-irreducible elements, limited only by the maximum lattice size. But in a distributive lattice we can limit their number as follows. We recall (see Corollary 112 in [10]) that the number of meet-irreducible elements in a distributive lattice equals the lattice length. As we build a lattice, we keep track of the meet-irreducible elements created so far, and at each level we compute an updated upper bound $M$ on the lattice length (based on the current length and the budget of remaining elements). The number of meet-irreducible elements, including the ones already created, is then limited to be at most $M$.

With this program, all distributive pieces and specials of $n \leq 60$ elements were generated (up to isomorphism), and classified with the method described in Section 4.1. Compositions were then counted using Theorem 1 . The results are shown in Table 2.

An exponential lower bound is derived in the same way as with modulars. Using Theorem 1 with the known numbers of distributive pieces of up to 60 elements, and plugging in zeros for larger pieces, we obtain lower bounds on $\mathrm{CF}_{n}, \mathrm{CS}_{n}$ and $\mathrm{CN}_{n}$ whose growth ratios settle a little above 1.7250 for $n$ large enough. To obtain rigorous lower bounds, we choose a convenient starting point $n=100$, convenient constant coefficients in front, and apply induction.

Theorem 5 The numbers of nonisomorphic distributive compositions of types CF, CS and $C N$ have the following lower bounds, when $n \geq 100$ :

$$
\begin{aligned}
\mathrm{CF}_{n} & \geq 0.010600 \times 1.7250^{n} \\
\mathrm{CS}_{n} & \geq 0.000092 \times 1.7250^{n} \\
\mathrm{CN}_{n} & \geq 0.001950 \times 1.7250^{n}
\end{aligned}
$$

Proof For $100 \leq n \leq 161$ the claim follows by direct calculation with Theorem 1, using the numbers of pieces from Table 2, and zeros when the number of pieces is not known. 


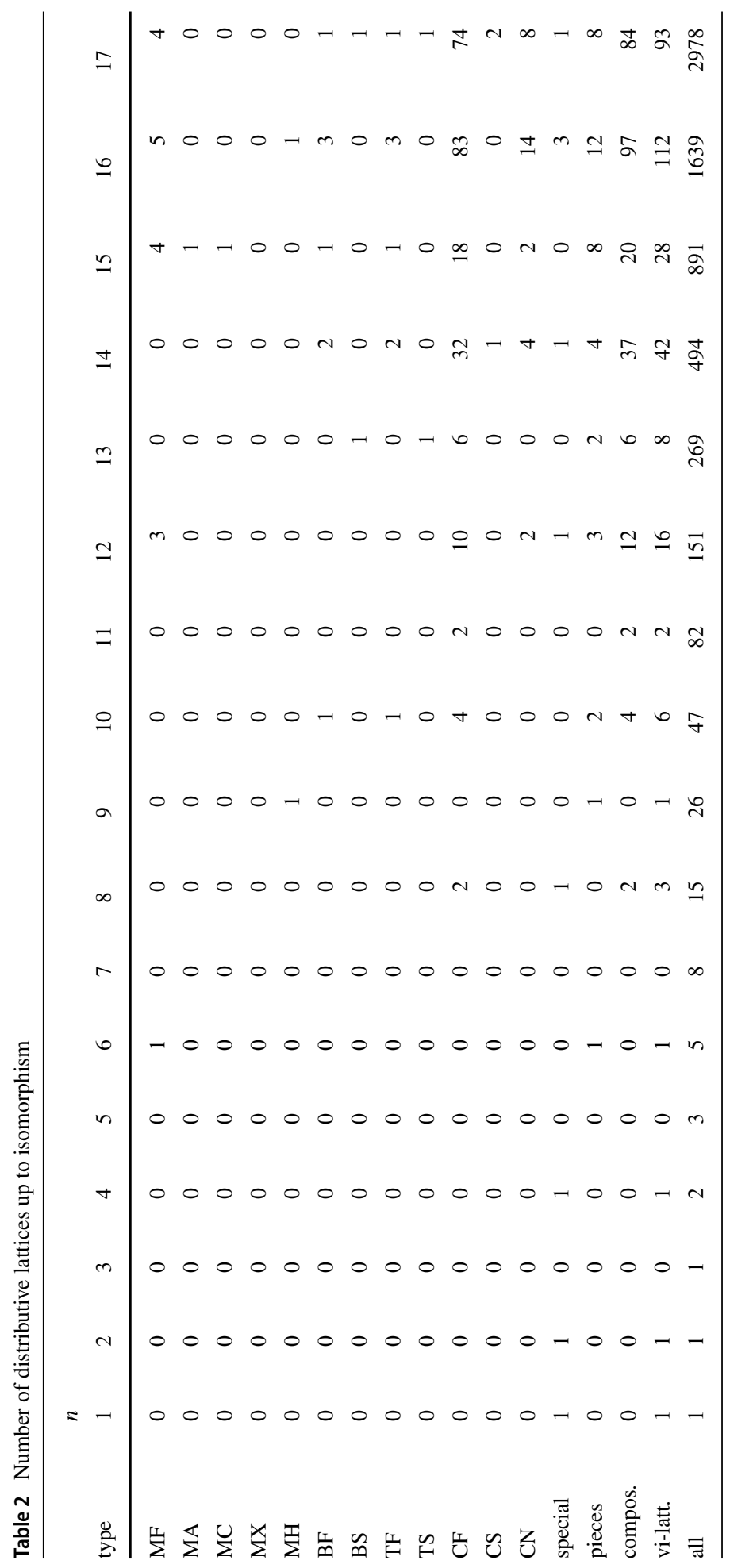




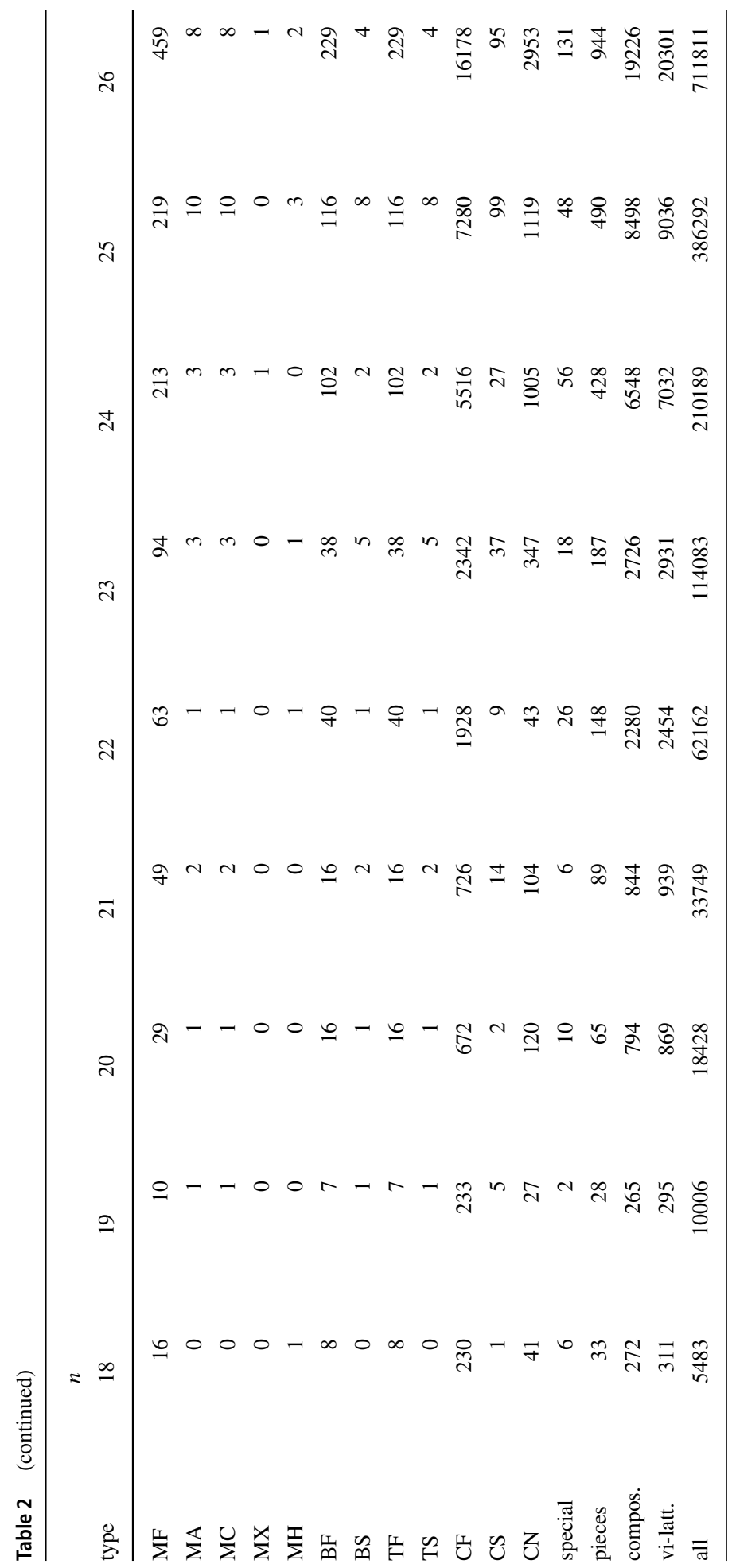




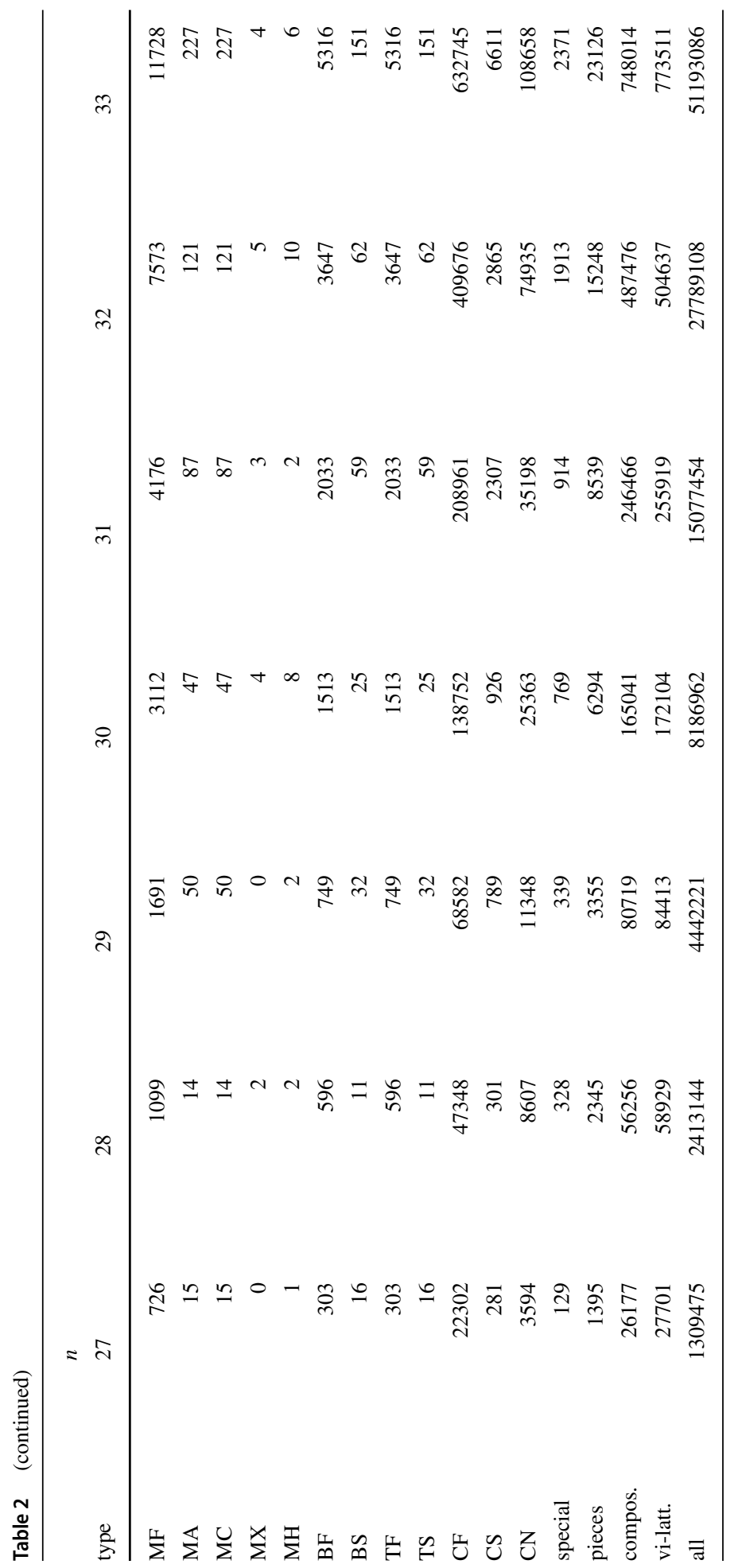




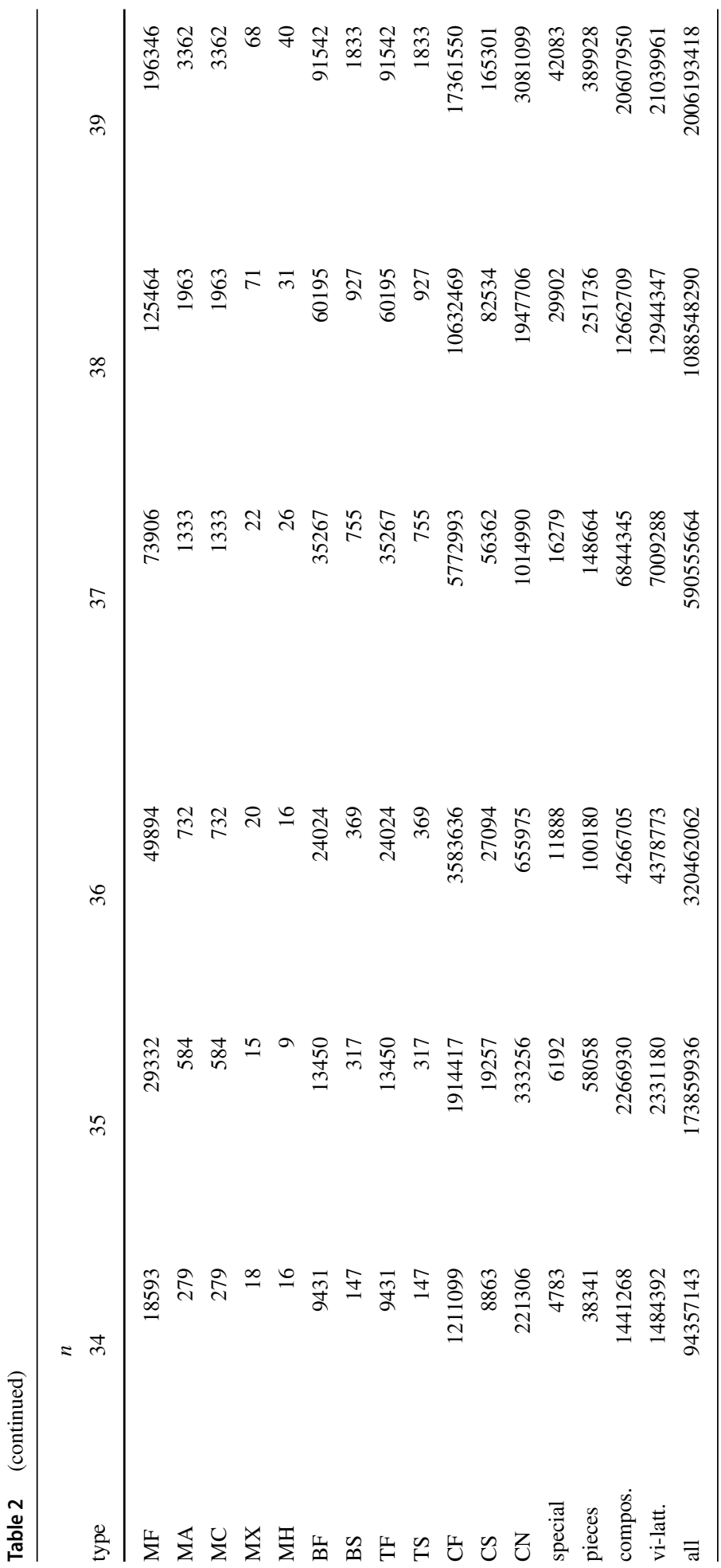




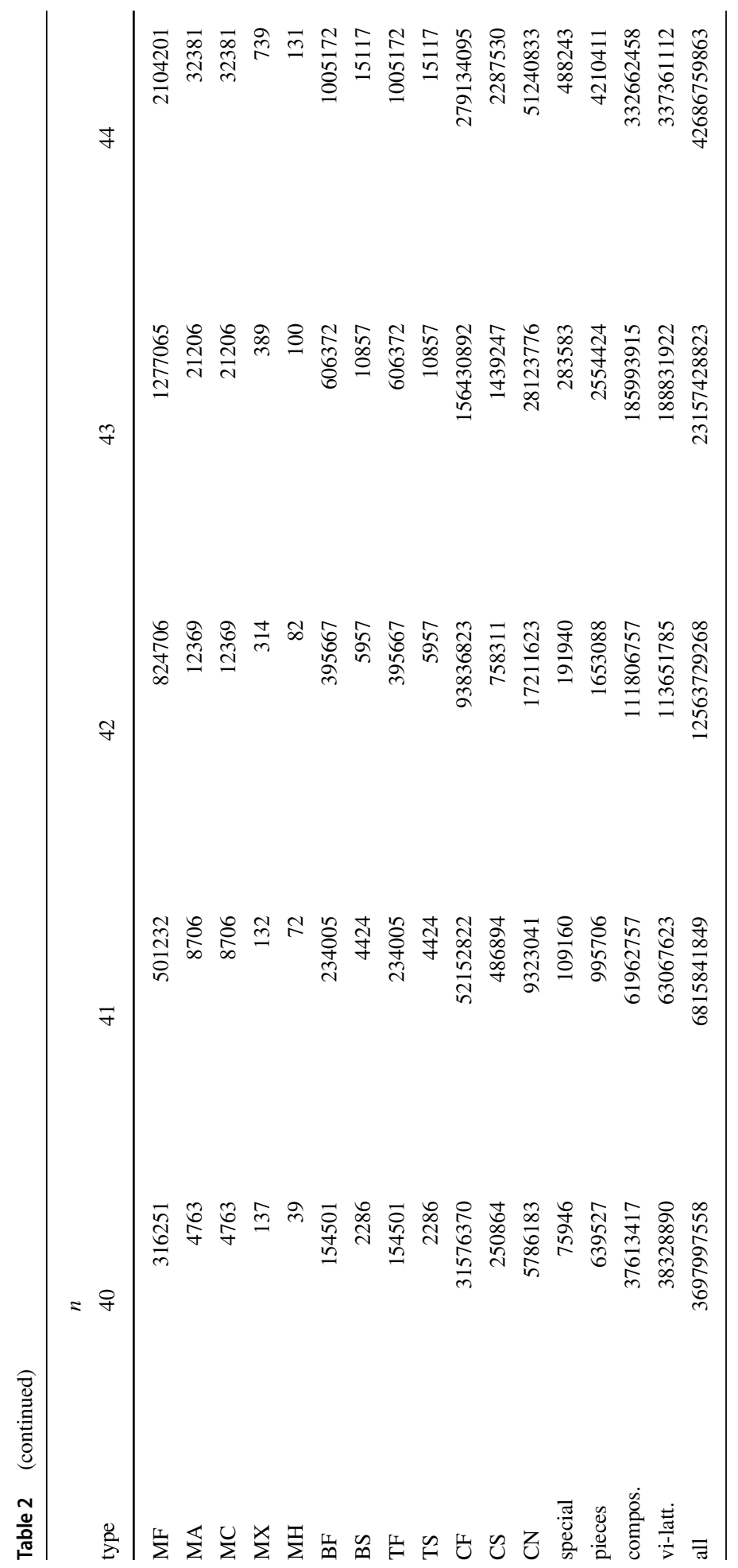




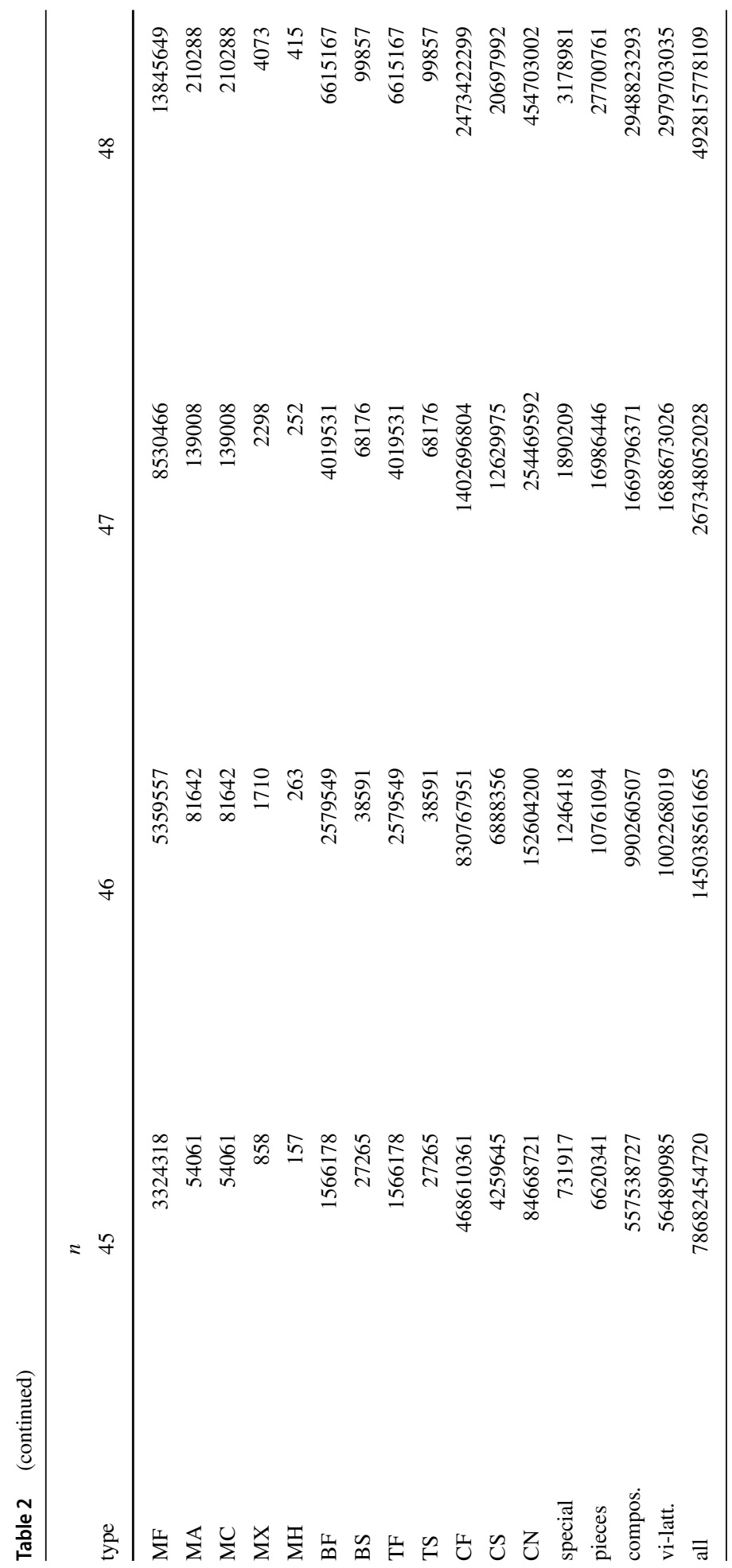




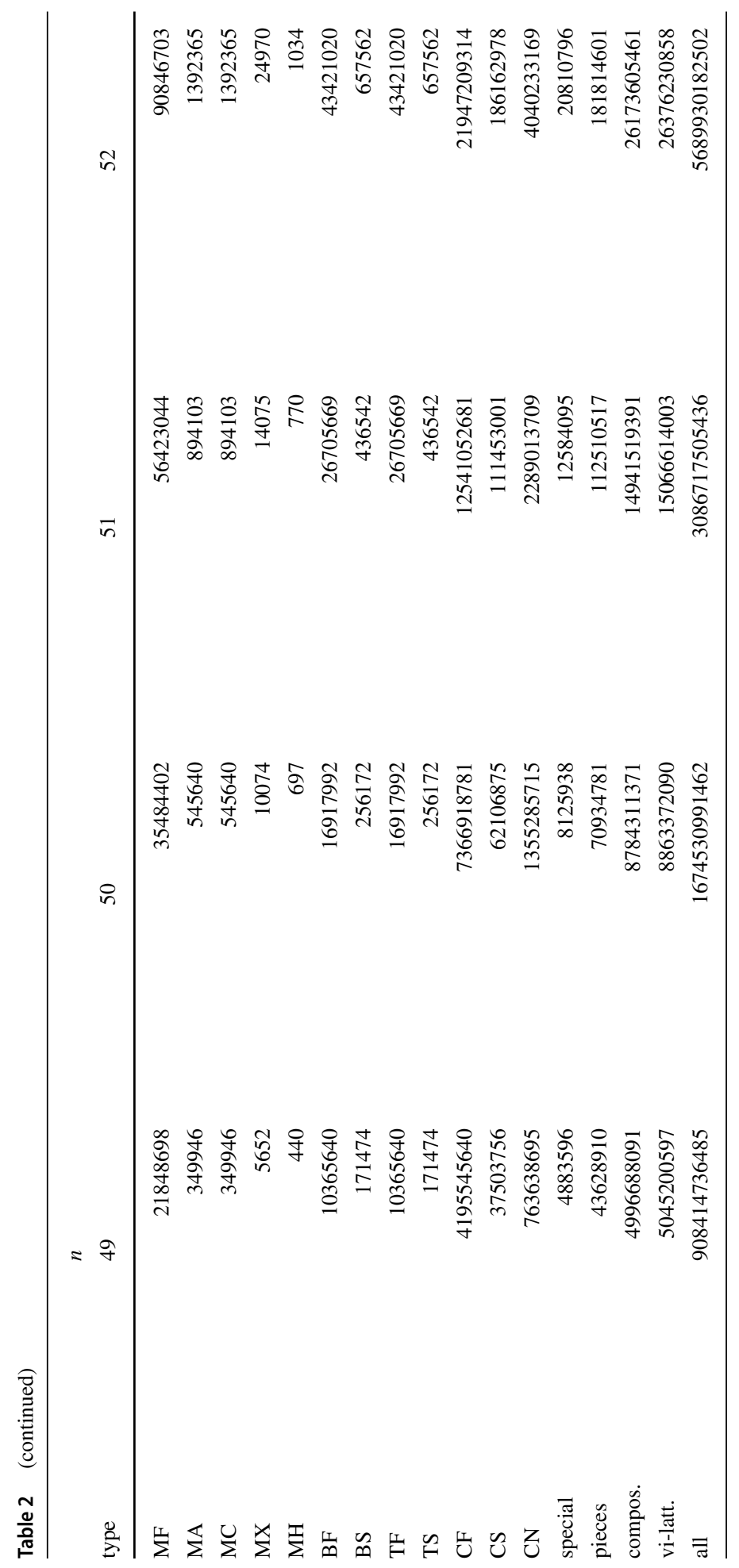




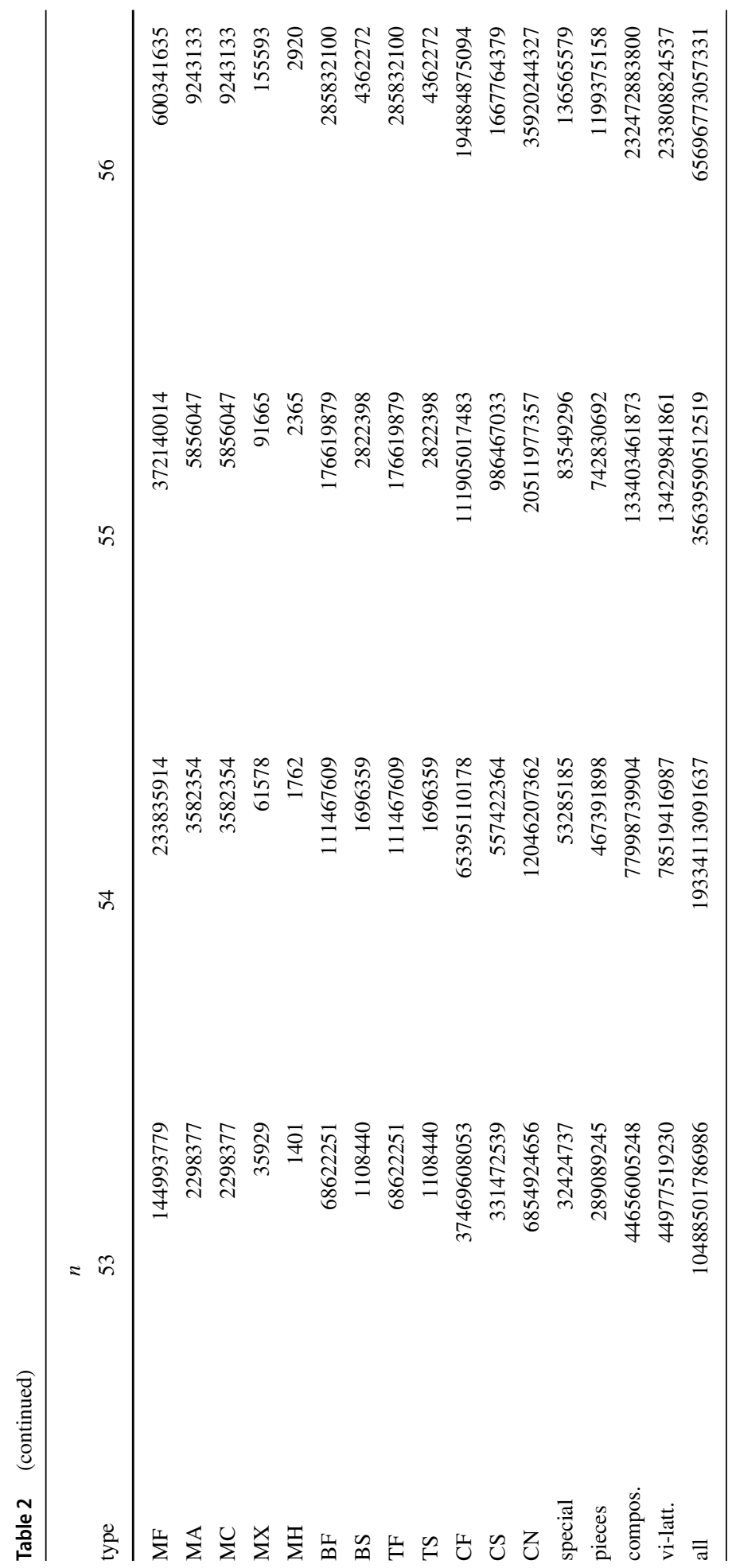




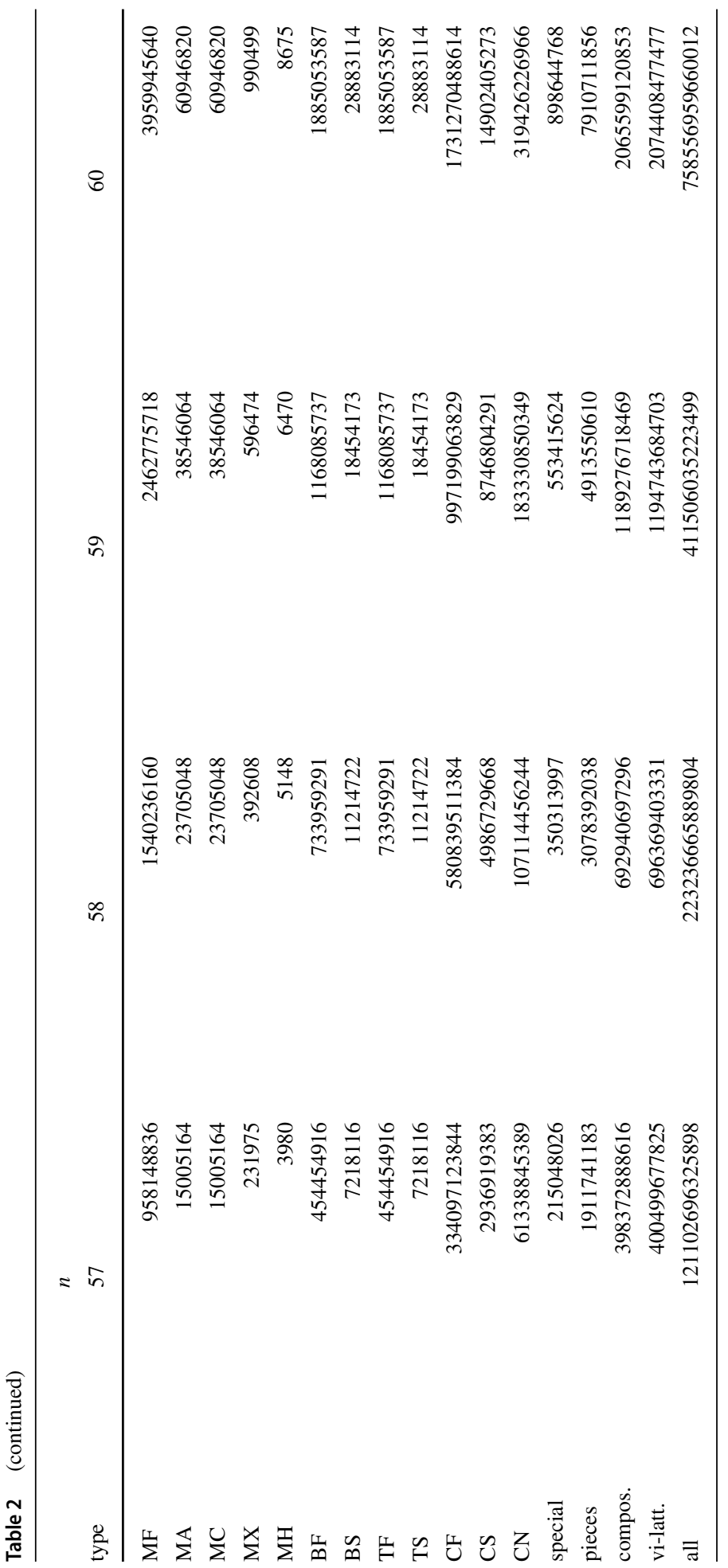


For $n>161$ the claim follows by induction on $n$. Let $n>161$ be arbitrary, and assume that the claimed lower bounds hold on $\mathrm{CF}_{m}, \mathrm{CS}_{m}$ and $\mathrm{CN}_{m}$ when $n-61 \leq m \leq n-1$. Then applying Theorem 1 gives the claimed lower bounds on $\mathrm{CF}_{n}, \mathrm{CS}_{n}$ and $\mathrm{CN}_{n}$, which completes the induction.

Corollary 2 There are at least $0.012642 \times 1.7250^{n}$ nonisomorphic distributive vi-lattices of $n$ elements when $n \geq 100$.

Proof Add up the three lower bounds from Theorem 5.

Theorem 6 There are at least $0.088 \times 1.8433^{n}$ nonisomorphic distributive lattices of $n$ elements when $n \geq 100$.

Proof For $100 \leq n \leq 400$ the claim follows by direct calculation. First use Theorem 1 to compute the exact numbers of distributive vi-lattices for $n \leq 60$, and lower bounds for $60<n \leq 400$ by replacing the unknown piece counts with zeros. Then apply Eq. 1 to compute lower bounds on distributive lattices.

For the induction step, let $n>400$ be arbitrary, and assume that the claimed lower bound holds for the previous 300 values. Applying Eq. 1 then gives the claimed lower bound for the number of $n$-element distributive lattices. This completes the induction.

The accompanying SageMath code [14] demonstrates how the lower bounds can be verified by calculation: see functions paper_lower_bound_verify_distributive and paper_v1_lower_bound_verify_distributive.

\subsection{Semimodular Lattices}

Although semimodular vi-lattices are 2-summable, vertical 2-sum is not very useful with them. For example, only about $23 \%$ of the 25 -element semimodular vi-lattices are compositions (from data in [16]). This is in stark contrast with modular and distributive lattices. Basically this is because semimodular lattices are short and wide (cf. Figures 4-5 in [5]). For this reason we do not include tables of semimodular lattices here, but such tables can be easily computed using the accompanying program code.

We could apply the same techniques as above to obtain an exponential lower bound. But an asymptotically stronger lower bound is obtained by constructing semimodular lattices from Steiner triple systems. A Steiner triple system is a set of $k$ elements (points) and a collection of their 3-sets (triples), such that each pair of distinct points occurs in exactly one triple. By counting the pairs it is easily seen that the number of triples must be $k(k-1) / 6$. It is known that Steiner triple systems on $k$ points exist if and only if $k \equiv 1$ or $3(\bmod 6)$; such values of $k$ are called admissible.

Given a Steiner triple system on $k \geq 7$ points, if we take the points as atoms, the triples as coatoms, and augment a top and a bottom, we obtain a rank-three semimodular vi-lattice, because each pair of atoms is covered by exactly one coatom. The lattice has $k+k(k-$ 1) $/ 6+2$ elements.

Theorem 7 For any $n \geq 100$, the number of nonisomorphic semimodular rank-three vilattices containing $n$ elements is at least

$$
\left(0.3286 n^{1 / 8}\right)^{n}
$$


Proof Let $n \geq 100$ be given. Choose the largest admissible $k$ such that

$$
k+k(k-1) / 6+2 \leq n .
$$

Then $k \geq 21$, and because admissible values are at most 4 units apart, we have

$$
n<(k+4)+(k+4)(k+3) / 6+2<k^{2} / 3 .
$$

Let $N$ be the number of nonisomorphic Steiner triple systems on $k$ points. By Wilson's Theorem 2 [17], we have

$$
N \geq\left(e^{-5} k\right)^{k^{2} / 12},
$$

and using the bound $k>\sqrt{3 n}$ from Eq. 2 we obtain

$$
N \geq\left(e^{-5} \cdot \sqrt{3 n}\right)^{n / 4}=\left(e^{-5 / 4} \cdot 3^{1 / 8} \cdot n^{1 / 8}\right)^{n} \geq\left(0.3286 n^{1 / 8}\right)^{n} .
$$

From these $N$ Steiner triple systems we obtain $N$ nonisomorphic semimodular rankthree vi-lattices that have $n^{\prime}=k+k(k-1) / 6+2$ elements. By our choice of $k$ we have $n^{\prime} \leq n$. To each lattice, add $n-n^{\prime}$ coatoms covering an arbitrarily chosen atom of the highest updegree. This makes the lattices have exactly $n$ elements, preserving semimodularity and nonisomorphism, so the claim follows.

The lower bound in Theorem 7 is very loose (it does not even exceed 1 until $n \approx 7000$ ), and is presented here in simple terms just to demonstrate the asymptotic behaviour, that the number of semimodular vi-lattices grows faster than any exponential in $n$. The bound might be improved in several ways, for example, by using Keevash's recent improvement on Wilson's lower bound [18].

\subsection{Notes on Computation}

The main computational load was in generating the pieces and specials. For the largest sizes this was parallelized by running the lattice-generating program until a predefined number of elements had been added. The search state at those points was saved to a file, and the remaining work was divided among worker processes.

For modular lattices of 33, 34 and 35 elements, this computation took 8.9, 23.7 and $63.1 \mathrm{cpu}$-core-days on Intel Xeon Gold 6230 processors (clock frequency varying but nominally $2.1 \mathrm{GHz}$ ), so empirically the running time scales roughly as $2.66^{n}$. For distributive lattices of 58, 59 and 60 elements, the computation took 6.3, 10.4 and $17.2 \mathrm{cpu}$-core-days, scaling roughly as $1.66^{n}$. This is better than would be possible by any algorithm that relies on explicit enumeration of distributive vi-lattices, because the number of such lattices grows at least as $\Omega\left(1.7250^{n}\right)$.

The optimization in Section 4.3 that made it possible to count distributive lattices up to 60 elements is just one example, out of many, where the speed of a combinatorial search is greatly affected by a simple, innocent-looking bounding condition. Here its implementation takes about a dozen lines of code (see the accompanying program code for details). But already at 30 elements, it speeds up the generation of distributive pieces and specials from 153 seconds to 0.4 seconds; and the savings ratio keeps improving as the lattices grow.

Unfortunately, it is not always easy to come up with conditions that actually have a great impact, and are also fast enough to compute during the search. Given the large existing theory of the structure of distributive and modular lattices, it is conceivable that our latticegenerating program could still be much improved by imposing some other, so far untried, bounding conditions. 


\subsection{Partial Verification}

We describe here some of the methods that were used to partially verify the correctness of the computational results.

The pieces and specials were generated and classified twice, on different computers. The counts and the actual lattice listings were verified to be identical by comparing their MD5 checksums. This would help against transient hardware and operational errors, but not against systematic errors in the program.

The number of MA pieces equals the number of MC pieces in each column of Tables 1 and 2. This is as it should, because such pieces are duals of each other. The same holds between $\mathrm{BF}$ and TF pieces, and between BS and TS pieces.

We also performed a more thorough duality check. The rank sequence of a graded lattice is the sequence of its level sizes from bottom to top. The rank sequences of a lattice and its dual are reverses of each other. In Fig. 1 the BF example piece has rank sequence $(1,3,2,1)$, and its dual, the TF example piece has $(1,2,3,1)$. We counted the occurrences of each combination of symmetry type and rank sequence in pieces and specials, and verified that the numbers match between the dual pairs (MF-MF, MA-MC, MX-MX, MH-MH, BF-TF, BS-TS, and special-special). For example, among all 60-element distributive MX pieces, there are 2137 whose rank sequence is $(1,2,3,4,6,5,4,4,4,4,4,4,3,3,3,3,2,1)$, and 2137 whose sequence is its reverse. If any of the lattice listings were accidentally truncated or corrupted, this would likely be detected as a mismatch. An error in the lattice-generating or classification logic would also have a good chance of causing a mismatch.

As a consistency check of the Cartesian counting logic, we directly generated, classified and counted all distributive vi-lattices of 50 elements including compositions. In each class, the count thus obtained matches the calculated count in Table 2. Generating these 50-element lattices took $12.6 \mathrm{cpu}$-core-days, about 70 times longer than with compositions excluded, nicely demonstrating the benefits of Cartesian counting.

Our totals on rows "vi-latt." and "all" agree with previously published numbers of modular lattices to 33 elements [5, 13] and distributive lattices to 49 elements [1] (except at $n=1$, because we regard the singleton as a vi-lattice). The previous countings are independent in the sense that they did not employ the vertical 2-sum.

\section{Concluding Remarks}

One of our stated goals was to create more compact lattice listings by leaving out all compositions (vertical 2-sums of smaller lattices). As seen in Tables 1 and 2, this was more successful with distributive lattices than with modulars. Compositions make up $79 \%$ of the modular vi-lattices of 35 elements, and $99.6 \%$ of the distributive vi-lattices of 60 elements.

The observed growths of modular and distributive lattice counts, to the extent that they are now known, are illustrated in Fig. 3. In modular vi-lattices our lower bound $\Omega\left(2.3122^{n}\right)$ seems loose; the observed ratios keep increasing, hinting possibly of a (very slightly) superexponential growth. We note that no exponential upper bound is currently known on the number of modular lattices. In distributive vi-lattices the observed ratios seem to be converging, and our lower bound $\Omega\left(1.7250^{n}\right)$ seems rather tight.

Erné et al. have shown an upper bound of $O\left(2.33^{n}\right)$ on nonisomorphic distributive vilattices [1]. Our improved lower bound $\Omega\left(2.3122^{n}\right)$ on nonisomorphic modular vi-lattices is still not enough to separate the growth rates of these two families. To close the gap there are different options. We could count modular pieces further. Empirically, adding one element 

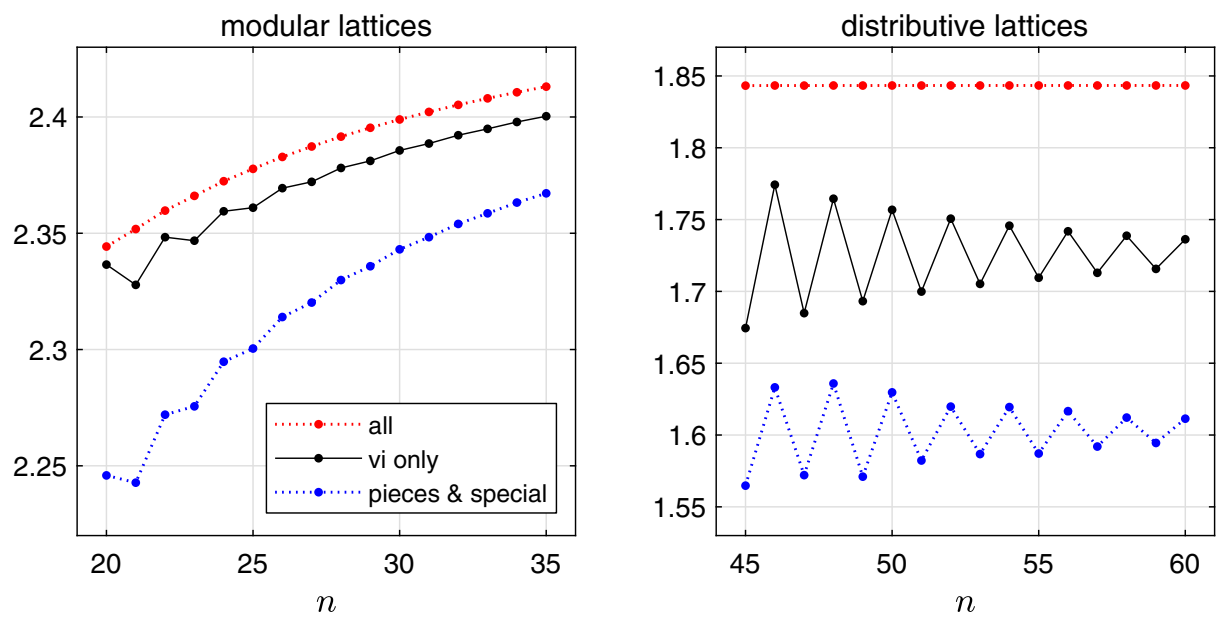

Fig. 3 Growth ratio of the number of nonisomorphic lattices, as lattice size increases from $n-1$ to $n$ elements

increases the base in our lower bound by about 0.0048 (diminishing as $n$ grows). Counting the pieces up to 40 elements would probably raise the lower bound above $\Omega\left(2.33^{n}\right)$. But this would take about $10000 \mathrm{cpu}$-core-days with the current lattice-generating program, and was deemed not worth the effort. Improving the algorithm or the bounding techniques might be a better idea. Another option is to improve the upper bound on distributive vi-lattices. Indeed, Erné et al. note that "with more effort" it could be improved considerably, at least to $2.28^{n}$. Combined with our lower bound, this would suffice to separate the growth rates.

Although our lower bounds are based on large computations, we must point out that proper analysis of symmetry is the key to good lower bounds. Indeed, using all our data on distributive lattices $(n \leq 60)$, if we ignore the symmetry cases (and lose the factors of 2 in Theorem 1), we only obtain a bound of $\Omega\left(1.6213^{n}\right)$ on distributive vi-lattices. In contrast, using just the distributive middle pieces of $n \leq 21$ elements (a truly modest collection of 134 lattices), our symmetry-distinguishing method already gives $\Omega\left(1.6818^{n}\right)$.

Acknowledgements The author wishes to thank the anonymous referee for helpful comments, and Peter Jipsen for suggesting the study of distributive lattices with the vertical 2-sum. Computational resources were provided by CSC - IT Center for Science and by the Aalto Science-IT project.

Funding Open access funding provided by Aalto University.

Data availability statement The data generated during this study are partially available in the EUDAT B2SHARE repository [19]. This contains the exhaustive listings of pieces and specials, up to sizes of 33 elements (modulars) and 55 elements (distributives). The data are stored in digraph6 format [11] and compressed with xz [20]. The larger lattices, modulars of 34-35 elements and distributives of 56-60 elements, are currently not available in EUDAT due to their size, but are available from the author on reasonable request.

Accompanying program code is available in Bitbucket [14]. This includes $C$ programs to generate the pieces and the specials, to classify and count them by symmetry type, and to perform the Cartesian counting. Also included is SageMath code for verifying the exponential lower bounds.

Open Access This article is licensed under a Creative Commons Attribution 4.0 International License, which permits use, sharing, adaptation, distribution and reproduction in any medium or format, as long as you give appropriate credit to the original author(s) and the source, provide a link to the Creative Commons licence, and indicate if changes were made. The images or other third party material in this article are included in the 
article's Creative Commons licence, unless indicated otherwise in a credit line to the material. If material is not included in the article's Creative Commons licence and your intended use is not permitted by statutory regulation or exceeds the permitted use, you will need to obtain permission directly from the copyright holder. To view a copy of this licence, visit http://creativecommons.org/licenses/by/4.0/.

\section{References}

1. Erné, M., Heitzig, J., Reinhold, J.: On the number of distributive lattices. Electron. J. Combin. 9, Article \#R24 (2002)

2. Gebhardt, V., Tawn, S.: Constructing unlabelled lattices. J. Algebra 545, 213-236 (2020)

3. Heitzig, J., Reinhold, J.: Counting finite lattices. Algebra Univ. 48, 43-53 (2002)

4. Jipsen, P., Lawless, N.: Generating all finite modular lattices of a given size. Algebra Univ. 74, 253-264 (2015)

5. Kohonen, J.: Generating modular lattices of up to 30 elements. Order 36, 423-435 (2018)

6. Kohonen, J.: Exponential lower bounds of lattice counts by vertical sum and 2-sum. Algebra Univ. 80 (2019)

7. Dowling, T.A., Wilson, R.M.: Whitney number inequalities for geometric lattices. Proc. Amer. Math. Soc. 47, 504-512 (1975)

8. Björner, A.: Shellable and Cohen-Macaulay partially ordered sets. Trans. Amer. Math. Soc. 260, 159183 (1980)

9. Collins, K.L.: Planar lattices are lexicographically shellable. Order 8, 375-381 (1992)

10. Grätzer, G.: Lattice theory: Foundation. Birkhäuser, Basel (2011)

11. McKay, B.D., Piperno, A.: Nauty and Traces home page. http://pallini.di.uniroma1.it/

12. McKay, B.D., Piperno, A.: Practical graph isomorphism, II. J. Symbolic Comput. 60, 94-112 (2014)

13. OEIS, the on-line encyclopedia of integer sequences. https://oeis.org/A006981. Number of unlabeled modular lattices with $\mathrm{n}$ elements

14. Kohonen, J.: Cartesian lattice counting. https://bitbucket.org/jkohonen/cartesian-lattice-counting/

15. OEIS, the on-line encyclopedia of integer sequences. https://oeis.org/A006982. Number of unlabeled distributive lattices with $n$ elements

16. Kohonen, J.: Lists of finite lattices (modular, semimodular, graded and geometric). http://doi.org/10. 23728/b2share.dbb096da4e364b5e9e37b982431f41de

17. Wilson, R.M.: Nonisomorphic Steiner triple systems. Math. Z. 135, 303-313 (1974)

18. Keevash, P.: Counting Steiner triple systems. In: European Congress of Mathematics, pp. 459-481 (2018)

19. Kohonen, J.: Modular and distributive lattices without vertical sums and 2-sums. http://doi.org/10.23728/ b2share.80c0b996508b4a7b8f9bb6b7919c492a

20. The Tukaani Project: XZ Utils. https://tukaani.org/xz/

Publisher's Note Springer Nature remains neutral with regard to jurisdictional claims in published maps and institutional affiliations. 